\title{
Leishmaniasis Recidivans in Rural Venezuela
}

\author{
Ariadna Perez Sanchez ${ }^{1}$, Rajani Katta ${ }^{2}$
}

1 Department of Internal Medicine, The University of Texas Health Science Center, San Antonio, TX, USA

2 Department of Dermatology, McGovern Medical School, Houston, TX, USA

Key words: leishmaniasis recidivans, Leishmania braziliensis, localized cutaneous leishmaniasis, ulceration, antimonials

Citation: Perez Sanchez A, Katta R. Leishmaniasis recidivans in rural Venezuela. Dermatol Pract Concept. 2020;10(3):e2020062. DOI: https://doi.org/10.5826/dpc.1003a62

Accepted: April 16, 2020; Published: June 29, 2020

Copyright: @2020 Perez Sanchez and Katta. This is an open-access article distributed under the terms of the Creative Commons Attribution License, which permits unrestricted use, distribution, and reproduction in any medium, provided the original author and source are credited.

Funding: None.

Competing interests: The authors have no conflicts of interest to disclose.

Authorship: Both authors have contributed significantly to this publication.

Corresponding author: Rajani Katta, MD, McGovern Medical School at UT Health Houston, 6800 West Loop South, Ste. \#180, Bellaire, TX 77401, USA. Email: info@kattamd.com

\section{Clinical Presentation}

A 66-year-old man presented with 2 asymptomatic ulcerations on the right cheek (Figure 1). These began as enlarging papules that ulcerated 2 weeks later. He had been treated for leishmaniasis a year previously, with an ulcer in the same location. Diagnosis had been made by skin biopsy and polymerase

Figure 1. Two ulcerations on the right cheek, overlying scar from previous infection.

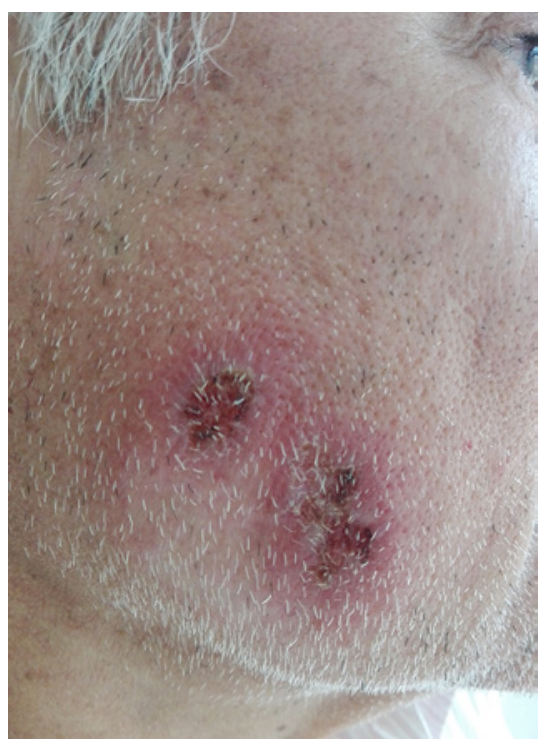

chain reaction, identifying Leishmania braziliensis. At that time, he was given intramuscular meglumine antimoniate; his lesions had healed completely within 1 month.

\section{Teaching Point}

Leishmaniasis recidivans (LR) is a rare presentation of localized cutaneous leishmaniasis, recurring at the site of a previously healed ulcer [1], as in this case. It typically affects the face, often the cheek. Leishmania braziliensis is one of the species linked to New World LR. While the pathogenesis of LR is not known, risk factors include parasite resistance and incomplete treatment. Resistance to antimonials is increasingly a concern in some regions [2].

\section{References}

1. Masood S, Naveed S, Alvi RU. Infiltrated leishmaniasis recidivans cutis on the face: a rare clinical presentation. Trop Doct. 2012;42(2):120-121. https://doi.org/10.1258/td.2011.110396

2. Ponte-Sucre A, Gamarro F, Dujardin JC, et al. Drug resistance and treatment failure in leishmaniasis: a 21st century challenge. $P L O S$ Negl Trop Dis. 2017;11(12):e0006052. https://doi.org/10.1371/ journal.pntd.0006052 